\title{
A 4D STEM-in-SEM Analysis of Hexagonal Boron Nitride
}

Jason Holm and Elisabeth Mansfield

NIST, Boulder, Colorado, United States

Hexagonal boron nitride ( $\mathrm{hBN}$ ) is an electrically insulating material of interest for two-dimensional (2D) materialsbased applications. Different synthesis processes for high quality hBN are well studied [1], and a common form of $\mathrm{hBN}$ is crystalline flakes comprised of van der Waals bonded monolayers. Because monolayers exfoliated from bulk-like flakes are generally of most interest for 2D applications, the flakes as a whole generally receive less attention. This is somewhat surprising because defects such as large, crisp wrinkles on the flakes can be easily observed (Fig. 1a). To that end, we use 4-dimensional (4D) scanning transmission electron microscopy in a scanning electron microscope (STEM-in-SEM) to examine a cross-sectional sample obtained from one of those wrinkles (Figure 1a, inset). Different approaches to visualizing the 4D dataset are used to show significant variation among hBN layers.

A focused $\mathrm{Ga}^{+}$ion beam was used to prepare a lift-out cross-section from a wrinkle similar to the one shown in Figure 1a. Because hBN is an insulator, the probe tip was maintained in close proximity to the lift-out location to mitigate charge buildup during milling. Figure $1 \mathrm{~b}$ shows a schematic of the 4D STEM-in-SEM experimental apparatus [2]. Here, a scintillator, mirror assembly, and CCD camera were used to image electrons forward scattered through the sample in a Zeiss Gemini 300 SEM. LabVIEW was used to synchronize the electron beam and the camera, and MATLAB was used for offline 4D dataset analyses [3].

Figure 1a (inset) shows a transmission image of the sample using a Zeiss aSTEM detector. A large void beneath the sample surface and stripes of different contrast intersecting at lines (twin boundaries) extending from the corners of the void are apparent. Figure 2a shows an annular dark-field (ADF) image of a smaller region including the void, and two diffraction patterns (DP) averaged over the specified areas, all obtained from a 4D dataset. The DP from the region indicated in red shows a ring suggestive of polycrystalline structure. The DP from the immediately adjacent region (indicated in green) shows a row of spots suggesting that this part of the sample is crystalline, with $\mathrm{BN}$ layers oriented normal to the direction of the row of spots.

Figures $2 b$ and $2 c$ show a slightly different region of the sample obtained using a different $4 \mathrm{D}$ dataset. The DP in Fig. $2 b$ shows the average diffraction pattern of the entire dataset with two virtual apertures indicated in green. The conventional BF image (Fig. 2b) was formed with the circular aperture, and the nonconventional DF image (Fig. 2c) was formed with the linear aperture. Structures appear fairly well-aligned with a few defects in the BF image, but the DF image shows widespread defects. Figure 2c also shows a single DP obtained from the spot shown in Fig. $2 b$, demonstrating that this region of the sample comprises a layered structure.

We have shown that 4D STEM can be implemented in an SEM and that the datasets enable conventional and nonconventional imaging conditions to be applied offline. To that end, we have shown that depending on the signals allowed to contribute to images, sample defects may not always be apparent in STEM-in-SEM images. The two images shown here, however, demonstrated that layered crystalline structure does exist in the flakes, and that these structures are interspersed with defects and non-crystalline regions throughout the sample. We have also demonstrated how 4D STEM-in-SEM can be applied to an insulating material that can be challenging to image with $30 \mathrm{keV}$ electrons in conventional SEM mode due to sample charging. 

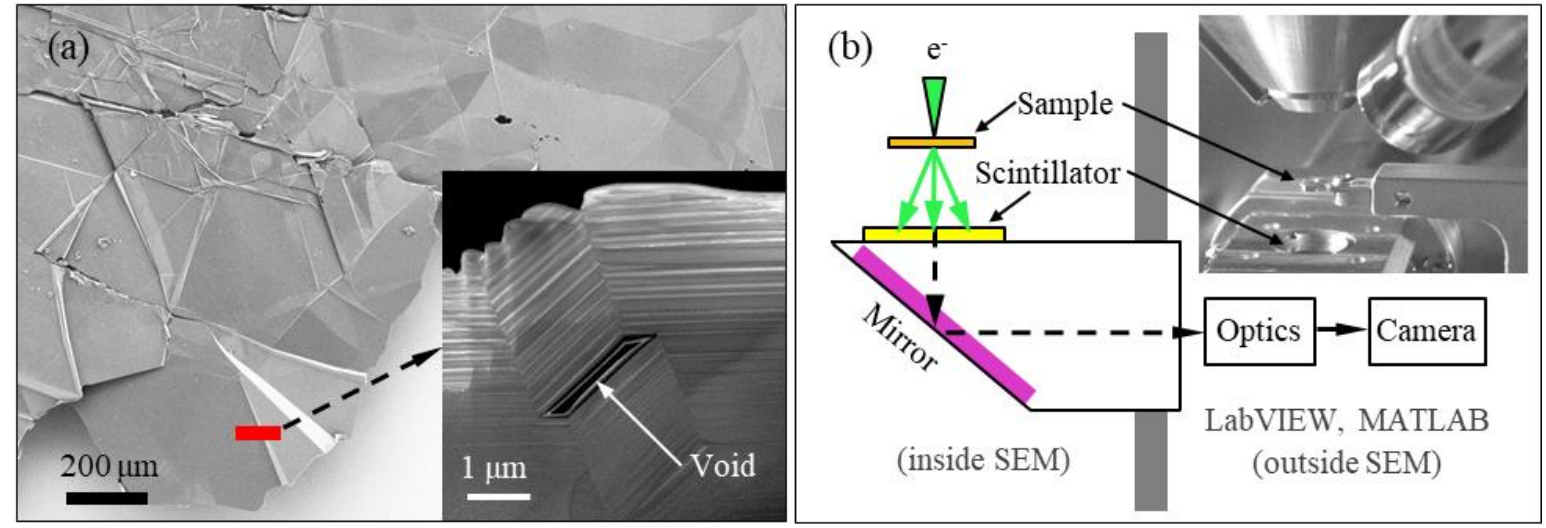

Figure 1. (a) Secondary electron image of a single hBN flake with surface wrinkles. The inset shows a FIB lift-out sample obtained from a location similar to the one indicated by the red line. (b) 4D STEM-in-SEM experimental apparatus schematic with an image showing the chamber interior.
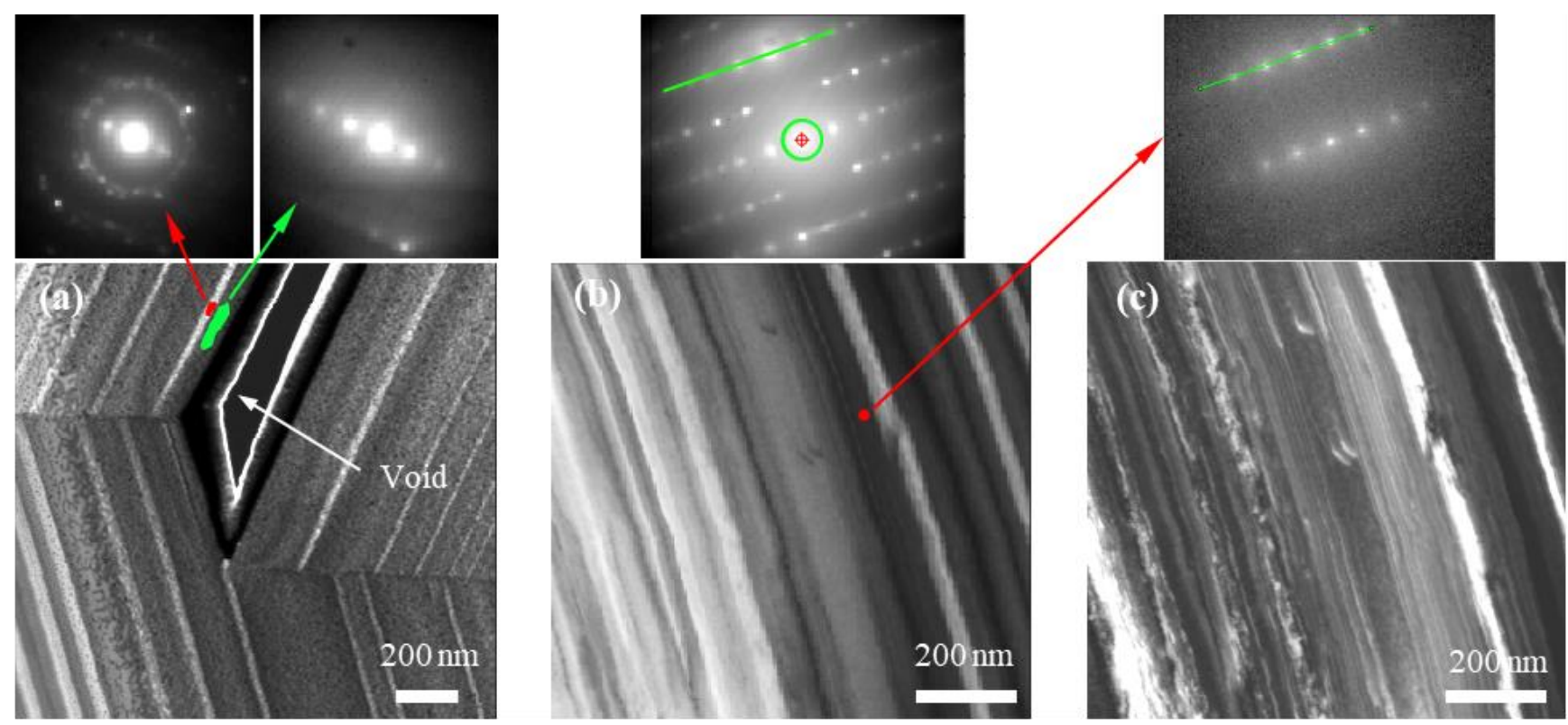

Figure 2. (a) Virtual ADF image (30 keV) showing part of the void and surrounding area located under the wrinkle. DPs were averaged over the indicated areas. (b) BF image showing layer alignment. The DP represents the average of all DPs in the 4D dataset, with virtual apertures outlined in green and the direct beam center mark indicated in red. (c) Nonconventional DF image (virtual aperture is the green line) highlighting regions with and without defects. The DP was obtained from the indicated location in (b).

\section{References}

[1] Zastro, M., Nature, 2019 (572) 429-432.

[2] Caplins, B. W., et al., Ultramicroscopy, 2020 (291) 113137.

[3] Trade names and company products identified in the text do not imply recommendation or endorsement by NIST, nor do they imply that the products are best suited for the purpose. 